\title{
DESARROLLO DE LA COMPETENCIA AMBIENTAL EN LOS ESTUDIANTES DE LA CARRERA DE INGENIERÍA INDUSTRIAL DE LA UNIVERSIDAD TECNOLÓGICA INDOAMÉRICA VÍCTOR HUGO ABRIL PORRAS
}

\author{
Dr. en Agricultura \\ Universidad Tecnológica Indoamérica (UTI) \\ Centro de Investigación, Innovación y Desarrollo (CIID) \\ c/ Bolívar 20-35 entre Quito y Guayaquil. Ambato - Ecuador \\ Telf: (593-3) 2421452 - (593-3) Fax: (593-3) 2421713 \\ victorabril@uti.edu.ec, vhabril@gmail.com
}

\section{Cómo citar este artículo:}

Abril, V. (2011). Desarrollo de la competencia ambiental en los estudiantes de la carrera de Ingeniería Industrial de la Universidad Tecnológica Indoamérica. Espiral, Revista de Docencia e Investigación. 1, (1), 67 - 72

\section{Resumen}

El presente trabajo conjuga la labor realizada por los

estudiantes de la Carrera de Ingeniería Industrial, en el proyecto formativo de "Gestión Ambiental". Los cuarenta y nueve estudiantes del noveno nivel, participaron conjuntamente con el docente en la realización de once trabajos de investigación sobre temas ambientales que coadyuvan al fortalecimiento de la educación ambiental. El objetivo es refexionar sobre modelos innovadores de gestión-transformación educativa que promueven el desarrollo sustentable. En tal virtud fueron diseñados estudios sobre contaminación ambiental, reciclaje y manejo de recursos naturales, como experiencias de casos reales que han generado soluciones prácticas y efcaces.

Palabras Clave: desarrollo sustentable, educación ambiental, impactos ambientales, medioambiente, proyecto de investigación.

\footnotetext{
Abstract

The actual job conjugates the work carried out by the students of the Career of Industrial Engineering, in the formative project of "Environmental Management". The forty nine students of the ninth level, they participated jointly with the educational one in the realization of eleven investigation works on environmental topics that cooperate to the invigoration of the environmentai education. The objective is the one
}

of meditating on innovative models of management - educational transformation that promote the sustainable development. In such a virtue studies have been designed it has more than enough environmen-

tal contamination, recycle and handling of natural resources, as experiences of real cases that have generated practical and effective solutions.

Key words: sustainable development, environmental education, environmental impacts, environment, project of investigation.

\section{Introducción}

El Plan Nacional de Educación Ambiental para la Educación Básica y el Bachillerato 2007 - 2016 de la República del Ecuador en su introducción incluye la frase idónea que

encierra todo el pensamiento referente a la línea de investigación "Educación Ambiental y Desarrollo Sostenible" del Centro de Estudios en Educación (CEE) y que es:

"Para lograr una educación de calidad es necesario un ambiente de calidad". 
El ámbito del desarrollo sostenible abarca a tres ámbitos: el ambiental, el económico y el social, conjugados a $\mathrm{f} n$ de satisfacer las necesidades de las generaciones presentes sin comprometer las posibilidades del futuro y atender sus propias necesidades.

Asimismo, el desarrollo y el bienestar social, están limitados por el nivel tecnológico, los recursos del entorno y la capacidad del medioambiente para absorber los efectos de la actividad humana. Ante esta situación, se plantea la posibilidad de mejorar la tecnología y la organización social de forma que la naturaleza pueda recuperarse al mismo ritmo que es afectado por la actividad humana.

El presente trabajo relata las experiencias adquiridas en el proceso de producción de conocimiento del módulo formativo de Gestión Ambiental que se imparte en el noveno semestre de la Facultad de Ingeniería Industrial modalidad semi-presencial, donde se elaboraron, ejecutaron y evaluaron actividades académicas que permitieron el desarrollo de la competencia ambiental y la construcción de conocimientos al interior de sus involucrados con la asistencia y colaboración de diferentes entidades y profesionales durante su formación.

Numerosos autores presentan a la educación ambiental, en todos los niveles y sectores de la sociedad, como la principal solución a los problemas ambientales. No es la única, ya que debe ser acompañada por otras medidas de corte económico, político, y tecnológico, entre otras. Sin embargo, la aplicación de planes y programas de educación ambiental en países avanzados no ha logrado modifcar el ritmo de deterioro del ambiente ni la degradación de los recursos naturales a nivel global. Otros especialistas reconocen que la educación es una estrategia fundamental para el cambio de actitudes y comportamientos de la sociedad. Frecuentemente, en estas propuestas, se piensa que el cambio mediante la educación será posible de la mano de las nuevas generaciones. Estas perspectivas permiten englobar algunas tendencias comunes en la evolución de la educación ambiental, pero existen tantas prácticas como concepciones acerca del ambiente (EL HERALDO, La Educación Ambiental como Política de Estado, 13 de abril del 2008, P. 9-A).

\section{Antecedentes}

A inicios del 2009 la Dirección de Desarrollo Humano y Cultura del Honorable Consejo Provincial de Tungurahua ubicada en la ciudad de Ambato - Ecuador, conjuntamente con la Organización Ecológica Wira-Purú y la Corporación Educativa Nueva Acrópolis aunaron esfuerzos e ideas para plantear proyectos de participación científca, académica, de educación y de capacitación sobre temas ambientales que coadyuven al fortalecimiento del ambiente. En tal sentido, fue delineado el proyecto denominado "Los Arquitectos del Mundo Verde".

Posteriormente, el Consejo de Investigación de la Universidad Tecnológica Indoamérica, procedió con la aprobación para la participación en el encuentro a favor del planeta, para lo cual se delegó como responsable académico al docente del módulo formativo de "Gestión Ambiental" para la coordinación de la elaboración de proyectos que representen a la institución. 
Fortalecer la conciencia ambiental, con miras a buscarr posibles soluciones inteligentes con la participación de la ciudadanía.
El evento académico fue considerado como un espacio de difusión de una nueva una cultura verde que promueva nuevas alternati-

vas de mejora a los ecosistemas; el propósito del mismo fue el fortalecer la conciencia ambiental, con miras a buscar posibles soluciones inteligentes con la participación de la ciudadanía.

\section{Bases generales}

Los organizadores del evento en acto público presentaron el cronograma, bases generales y específcas, para los ocho (8) ejes temáticos:

- Audiovisuales (documentales, cortometrajes, películas, otros similares y afnes)

- Conferencias y talleres (ponencias)

- Exposiciones de arte en reciclaje

- Exposiciones del mundo natural (acciones realizadas en el terreno)

- Jardines

- Muestras científcas / tecnología verde.

- Proyectos orgánicos caseros

- Representaciones artísticas

\section{Metodología}

El mecanismo de participación como estrategia metodológica y pedagógica en el avance del conocimiento y la exploración ecológica fue desarrollado por el docente del módulo formativo de Gestión Ambiental conjuntamente con sus educandos en las horas clase normales durante tres (3) fnes

de semana. Se realizó por separado, pero simultáneamente en dos (2) paralelos con un total de cuarenta y nueve (49) estudiantes.

Primeramente cada alumno independientemente se inclinó por uno (1) de los ocho (8) ejes temáticos que desarrolle sus potencialidades, dinamismo, comunicación y, sobre todo, su experiencia laboral ya que estudian en la modalidad semi-presencial y en su gran mayoría trabajan de lunes a viernes en empresas relacionadas con el área industrial. Posteriormente se conformaron grupos de trabajo por afnidad de ideas que no sobrepasen los cinco (5) integrantes incluido un coordinadorlíder de equipo nominado de entre sus integrantes.

Posteriormente cada equipo desarrolló una posible idea al unifcar sus criterios en un solo trabajo grupal participativo habida cuenta las bases específcas, tiempos, recursos necesarios y que al mismo tiempo incluya conocimientos previos y adquiridos en el módulo formativo de Gestión Ambiental. Para esto utilizaron el formato institucional de elaboración de proyectos del Centro de Investigación, Innovación y Desarrollo de la UTI, a fn de tener un documento idóneo que permita desenvolverse bajo los mismos parámetros en forma homogénea.

Durante el proceso de tutorías los distintos grupos recibieron apoyo y directrices por parte del docente encargado vía internet mediante la plataforma institucional en el submenú Campus Virtual (http:/ / www.indoamerica.edu.ec/home.html). De esta manera se obtuvieron en total once (11) trabajos grupales. Para fniquitar la labor, cada grupo presentó su trabajo y productos desarrollados con su respectivo soporte magnético.

La evaluación se realizó con los siguientes parámetros: proyecto escrito, tríptico in- 
formativo y video. El jurado califcador estuvo conformado por un integrante de cada grupo a $\mathrm{fn}$ de seleccionar los tres (3) mejores trabajos por curso. El docente no intervino en ninguna deliberación, tan sólo como mediador del proceso.

Seguidamente, se reunieron los representantes de seis trabajos preseleccionados (tres por curso) para realizar la misma tarea refnadora y obtener un solo veredicto por nivel. De esta manera se alcanzó tener los tres trabajos fnales que participarían en el concurso.

Finalmente, el docente ejecutó los registros respectivos de inscripción en las fchas ofciales que incluían: datos institucionales y de representación, área de participación, categoría, proyecto físico y respaldo magnético, con frmas de responsabilidad.

\section{Resultados}

A fnales de noviembre del 2009 se dio cumplimiento al programa de cierre y premiación del encuentro, en donde se reconoció a la Universidad Tecnológica Indoamérica por la contribución académico-científca en el programa ambiental promovido por el Gobierno Provincial con la entrega de una placa de agradecimiento por la valiosa participación y aporte en el Primer Encuentro de los Arquitectos del Mundo Verde en el área de Ciencia y Tecnología Verde. Ver link: http://uti-revistavirtual.blogspot.com/search/label/Reconocimiento\%20de $\% 20$ arquitectos $\% 20$ del $\% 20$ mundo\%20verde

Otro trabajo estudiantil que no se incluyó en este concurso fue modifcado y adaptado

para ser presentado en el Primer Congreso

Ecuatoriano de Gestión Ambiental Urbana y Expoambiental Urbana, organizado por la Universidad Técnica Particular de Loja (UTPL) y Fundación Natura del Ecuador. El Congreso tuvo como fnalidad reunir a diferentes actores para compartir experiencias y aportes científco-técnicos y de gestión, intercambiar resultados, debatir estrategias efectivas para el desarrollo sostenible de las ciudades; además, promover la conformación de una red de instituciones y personas relacionadas con la gestión ambiental urbana en el Ecuador. Ver link: http:// uti-revistavirtual.blogspot.com/search/label/ Gesti\%C3\%B3n\% 20Ambiental

Un último trabajo de aula que tampoco se incluyó en el concurso también fue retocado y acondicionado para ser presentado en el IV Congreso Nacional de Estudiantes de Ingeniería Ambiental (CONEIA), organizado por la Facultad de Ingeniería Química y la Escuela Profesional de Ingeniería Ambiental de la Universidad Nacional San Luis Gonzaga de Ica - Perú. El Congreso se manejó bajo el lema - Planteando soluciones ecoefcientes con responsabilidad y compromiso ambiental. Ver link:http://uti-revistavirtual. blogspot.com/search/label/

Desarrollo sustentable, se constituye al momento, en la mejor alternativa para generar los cambios sociales para mejorar la calidad de vida, la rentabilidad económica y el uso sustentable de los bienes y recuirsos naturales
Participaci\% C3\%B3n\%20Internacional

En la actualidad el docente del módulo formativo de Gestión Ambiental continúa con sus labores con diferentes grupos de la Facultad de Ingeniería Industrial de la Universidad Tecnológica Indoamérica con los cuales ha conseguido resultados que han sido presentados en diversos eventos académicos a nivel nacional e interna- 
cional siempre con la participación de sus estudiantes. Ver link: http://uti-revistavirtual. blogspot.com/search/label/Gesti\%C3\%B3n\%20 Ambiental\%20y\%20Ecol\%C3\%B3gica medidas de precautelación del entorno, la contaminación en general y el recalentamiento del Planeta, entre otros. Ante esta realidad es urgente que las instituciones educativas de todo nivel, más aún la Universidad, enseñe a los estudiantes el manejo de los recursos naturales con sentido de racionalización y protección del medio ambiente [3] Carrión.

Es necesario tomar conciencia ciudadana y crear hábitos de conservación que permitan mantener una efciente armonía en cada uno de los elementos de nuestro medio ambiente. A partir básicamente de un trabajo teóricopráctico, totalmente independiente del tablero y la tiza, es decir, una ecología de campo de desarrollo comunitario y humano (...). La educación, es la llamada a generar cambios profundos en las sociedades en la formación de las futuras generaciones (...). Los acercamientos teóricos y prácticos con una dimensión educativa permiten de manera recreativa, colectiva e investigativa adquirir los conocimientos mínimos que todo ser humano debe tener con relación al medio ambiente. Induce a las personas en todos los niveles de la educación formal y no formal a generar prácticas positivas frente a su entorno natural. [4].

\section{Referencias Bibliográfcas}

Consecuentemente la asignatura de Educación Ambiental es de una relevancia y actualidad indiscutibles, ya que trata los principales problemas ecológicos del mundo, esto es: el desgaste inconsciente e incontenible de la naturaleza, el impacto del gigantesco desarrollo industrial, sin
Swisscontact. Guía Metodológica para la Incorporación del Enfoque de Medio Ambiente en los Programas de Formación Profesional Compartida. Quito - Ecuador: Cemaprimes Cía. Ltda., 2003, p. 5.

Ecociencia, Fundación Ecuatoriana de Estudios Ecológicos. Planifcación de la Gestión Ambiental y Diseño de Proyectos. Programa de capacitación para la gestión 
democrática de los recursos naturales. Proyecto fortalecimiento a gobiernos locales - Ecociencia. Quito -

Ecuador: Programa Parque en Peligro, 2006, p. 7.

Carrión, J. (1996) Educación Ambiental. Ecuador: Universidad Particular de Loja, p. 5.

Ríos, J. (2000) Ecología y Desarrollo Humano - un enfoque pedagógico ambiental. Bogotá: Cooperativa Editorial Magisterio. 